Communications in Physics, Vol. 28, No. 4 (2018), pp. 287-300

DOI:10.15625/0868-3166/28/4/12940

\title{
RAMAN LASER WITH A SINGLY RESONANT CAVITY: THEORETICAL MODEL AND EXPERIMENT
}

\author{
S. VOITIKOV ${ }^{1, \dagger}$, R. CHULKOV ${ }^{1}$, V. MARKEVICH ${ }^{1}$, V. ORLOVICH ${ }^{1}$, DO QUOC KHANH ${ }^{2}$, \\ NGUYEN XUAN TU ${ }^{2}$ AND PHAM HONG MINH ${ }^{2}$ \\ ${ }^{1}$ B.I. Stepanov Institute of Physics, 220072, Nezavisimosti ave. 68-2, Minsk, Belarus \\ ${ }^{2}$ Institute of Physics, Vietnam Academy of Science and Technology, 18 Hoang Quoc Viet Rd., Cau \\ Giay Distr., Hanoi, Vietnam \\ ${ }^{\dagger}$ E-mail: s.voitikov@dragon.bas-net.by
}

Received 08 August 2018

Accepted for publication 23 November 2018

Published 15 December 2018

\begin{abstract}
A semiclassical theoretical model of a Raman laser possessing a cavity which is resonant at the Stokes wavelength and not resonant at the pump wavelength has been developed. The wave equations for describing evolution of amplitudes of the pump and Stokes pulses in the Raman cavity have been derived. Results of the theoretical modeling have been analyzed for the conditions of an experiment with the Raman laser on barium nitrate crystal under the nanosecond pulse pump at $532 \mathrm{~nm}$. Numerically calculated energy characteristics of the Raman laser as well as space-time dynamics of the Stokes generation have been demonstrated to be in reasonable agreement with the experiment.
\end{abstract}

Keywords: stimulated Raman scattering, solid-state laser, external cavity Raman laser.

Classification numbers: 42.55.Ye, 42.65.Dr, 42.60.Jf.

(C)2018 Vietnam Academy of Science and Technology 


\section{INTRODUCTION}

The effect of stimulated Raman scattering (SRS) is known to be widely used for downfrequency conversion [1,2], pulse compression [3], improvement of beam quality (the Raman beam clean-up [4]) and spectral contrast [5] of laser radiation. Raman conversion has been successfully demonstrated under the time scales ranging from continuous-wave regime [6] to femtoseconds [7]. In many respects, this became possible owing to new synthesized Raman crystals which allowed new generation of compact and efficient Raman converters. Raman lasers which occupy a separate niche of such light converters propose a unique solution providing an enhanced degree of spectral selectivity and enabling low-divergent fluxes of the generated radiation.

A concept of Raman lasing suggests two implementation ways where the Raman crystal can be placed inside either one common cavity with the pump source or a separate cavity. The first option is often referred to intra-cavity Raman lasers (ICRLs) while the second one is known as external-cavity Raman lasers (ECRLs). Theoretical description of ICRLs has been developed earlier [8]. In this paper we have focused on ECRL's modeling. We have been motivated by the fact that such a ECRS laser scheme reveals some principal advantages over ICRL. One of them is its ability to control independently the fluxes of generated radiation. In addition, the threshold of Raman generation can be sufficiently reduced and the conversion efficiency to Stokes radiation can be enhanced considerably in the ECRL possessing a double-pass pump configuration.

Below, we describe a semiclassical theoretical model of the pulsed ECRL which considers Stokes generation in a singly resonant Raman laser cavity, i.e., this cavity is only resonant at the Stokes wavelength. Specifically, the model comprises the modified wave equations describing evolution of amplitudes of the Stokes and pump pulses in the Raman laser cavity. In our analysis, we adhere to the paraxial approximation as well as the rotating wave and slowly varying envelope approximations. We apply this model to study energy characteristics and space-time Stokes generation dynamics of the pulsed ECRL on barium nitrate $\left(\mathrm{Ba}\left(\mathrm{NO}_{3}\right)_{2}\right)$ crystal which is pumped by the nanosecond pulses at $532 \mathrm{~nm}$ from an actively Q-switched frequency-doubled Nd:YAG laser.

\section{MODEL}

In the proposed theoretical development, an axisymmetric ECRL with external axisymmetric optical pump is considered. The field in the laser cavity is formed by the traveling wave of external laser pumping, $F_{\text {pump }}(z, r, t)$, backward wave, $F_{\text {backward }}(z, r, t)$, (if any), and Stokes standing $T E M_{00}$ mode generated in the resonator, $E_{\text {Stokes }}(z, r, t)$, where $z$ and $r$ is longitudinal coordinate and transverse radius, respectively, $t$ is time. In the framework of the approximations of slowly varying amplitudes and rotating waves $[9,10]$, the field of forward and backward traveling pump waves (for example, the pump wave reflected back into the Raman crystal by the output mirror) is represented in the form

$$
\begin{gathered}
F_{\text {pump }}(z, r, t)=\frac{1}{2}\left[F_{p}(z, r, t) e^{-i \omega_{p} t} e^{i k_{p} \mu_{p} z}+c . c\right], \\
F_{\text {backward }}(z, r, t)=\frac{1}{2}\left[F_{b}(z, r, t) e^{-i \omega_{p} t} e^{-i k_{p} \mu_{p} z}+c . c\right],
\end{gathered}
$$

and the field of the Stokes $T E M_{00}$ standing wave, $E_{\text {Stokes }}(z, r, t)$, is represented in the form:

$$
E_{\text {Stokes }}(z, r, t)=\frac{1}{2}\left(E_{S}(t) e^{-i \omega_{S} t}+c . c .\right) U_{S}(z, r),
$$


where $F_{p}(z, r, t), F_{b}(z, r, t)$ and $E_{S}(t)$ are slowly varying amplitudes of the traveling forward and backward pump waves, and the amplitude of the Stokes standing wave, respectively, $\omega_{p}$ is the pump frequency, $\mu_{p}$ and $k_{p}$ are the refractive index and the wave number of the pump field in the Raman medium, respectively, $\omega_{S}$ is the Stokes frequency, $U_{S}(z, r)$ is the normalized spatial distribution of the Stokes standing wave. The Gaussian-like Stokes standing wave in a cavity with a Raman crystal with antireflective coatings reads as follows [11]:

$$
\begin{gathered}
U_{S}(r, z)=\sqrt{\frac{2}{L_{S}}}\left\{\frac{1}{\sqrt{\mu_{S}^{(m)}}} u_{S}^{(m)}(r, z), z_{1}^{(m)}<z<z_{2}^{(m)}\right\}, \\
u_{S}^{(m)}(r, z)=\sqrt{\frac{2}{\pi r_{0}^{2}}} \exp \left(-\frac{r^{2}}{r_{0}^{2}}\right) \times \sin \left[k_{S}^{(m)}\left(z-z_{0}\right)\right],
\end{gathered}
$$

where subscript $m \in\{R, v\}, v$ and $R$ denote wave propagation in either the free space or Raman medium, respectively, $k_{S}^{(m)}$ are the corresponding Stokes wave numbers, $r_{0}$ is the waist radius of the of the Stokes mode, $z_{1}$ and $z_{2}$ are the longitudinal coordinates of the Raman crystal ends, $L_{S}=$ $\sum_{m} \mu_{S}^{(m)} L^{(m)}$ is the optical path length of the cavity at the wavelength $\lambda_{S}, L^{(m)}$ are the propagation distances, $\sqrt{2 / L_{S}}$ is the normalization factor, $\mu_{S}^{(m)}$ is a refractive index of a Raman medium. In Eq. (4), the factor $\frac{1}{\sqrt{\mu_{S}^{(m)}}}$ in the curly brackets ensures equality of the electromagnetic field fluxes between the gaps and adjacent laser elements with antireflection coatings. The normalization in Eq. (4) is chosen so that the Stokes energy $W_{S}(t)$ in the Raman cavity is expressed in terms of the field amplitude without any spatially dependent factors [11], namely,

$$
\begin{gathered}
W_{S}(t)=\int_{V_{c a v}} \frac{\mu_{S}^{2}(z)}{4 \pi}\left|E_{S}(\vec{r}, t)\right|^{2} d x d y d z= \\
E_{S}^{*}(t) E_{S}(t) \int_{V_{c a v}} \frac{1}{8 \pi} \mu_{S}^{2}(z) U_{S}^{2}(r, z) d x d y d z=\frac{1}{8 \pi} E_{S}^{*}(t) E_{S}(t),
\end{gathered}
$$

where $V_{\text {cav }}$ is the cavity volume, $\mu_{S}(z)=\mu_{S}^{(R)}=\mu_{S^{-}}$for $z$ in the Raman crystal length range, and $\mu_{S}(z)=\mu_{S}^{(v)}=1$ - for $z$ inside the gaps between the cavity mirrors and the Raman crystal.

The wave equation for the fields in the Raman cavity has the following form according to the references $[9,10]$ :

$$
\begin{gathered}
\nabla^{2}\left[F_{\text {pump }}(z, r, t)=F_{\text {backward }}(z, r, t)+E_{\text {Stokes }}(z, r, t)\right]- \\
\frac{1}{c^{2}} \frac{\partial^{2}}{\partial t^{2}}\left[F_{\text {pump }}(z, r, t)+F_{\text {backward }}(z, r, t)+E_{\text {Stokes }}(z, r, t)+4 \pi P_{\text {lin }}(z, r, t)\right]=\frac{4 \pi}{c^{2}} \frac{\partial^{2}}{\partial t^{2}} P(z, r, t),
\end{gathered}
$$

where $P_{\text {lin }}(z, r, t)$ and $P(z, r, t)$ are the linear and nonlinear induced polarizations in the Raman medium, respectively. According to $[9,10]$, linear polarization can be written as follows

$$
P_{\text {lin }}(z, r, t)=\alpha_{p} F_{\text {pump }}(z, r, t)+\alpha_{p} F_{\text {backward }}(z, r, t)+\alpha_{S} E_{\text {Stokes }}(z, r, t),
$$

where $\alpha_{p}$ and $\alpha_{S}$ the Raman polarizability at the pump and Stokes wavelengths, respectively, which determine corresponding refractive indices to be $1+4 \pi \alpha_{p}=\mu_{p}^{2}$ and $1+4 \pi \alpha_{S}=\mu_{S}^{2}[9,10]$. The paraxial, rotating wave, and slowly varying envelope approximations allow transformation of the terms in wave equation (6) with longitudinally spatial and time derivatives of the second order into the terms with first-order spatial and time derivatives $[9,10]$. As a result of such a 
transformation, the terms with the spatial and temporal derivatives of the pump and Stokes waves take the form:

$$
\begin{aligned}
& \nabla^{2} F_{\text {pump }}(z, r, t)-\frac{1}{c^{2}} \frac{\partial^{2}}{\partial t^{2}} F_{\text {pump }}(z, r, t) \\
= & \frac{1}{2}\left(\frac{\partial^{2}}{\partial x^{2}}+\frac{\partial^{2}}{\partial y^{2}}\right)\left[\frac{1}{2} F_{p}(z, r, t) e^{-i \omega_{p} t} e^{i k_{p} \mu_{p} z}+c . c .\right] \\
& +\left[i \omega_{p}\left(\frac{\mu_{p}}{c} \frac{\partial}{\partial z}+\frac{\partial}{\partial t}\right) F_{p}(z, r, t) e^{-i \omega_{p} t} e^{i k_{p} \mu_{p} z}+c . c .\right], \\
& \nabla^{2} F_{b a c k w a r d}(z, r, t)-\frac{1}{c^{2}} \frac{\partial^{2}}{\partial t^{2}} F_{b a c k w a r d}(z, r, t) \\
= & \frac{1}{2}\left(\frac{\partial^{2}}{\partial x^{2}}+\frac{\partial^{2}}{\partial y^{2}}\right)\left[\frac{1}{2} F_{b}(z, r, t) e^{-i \omega_{p} t} e^{i k_{p} \mu_{P} z}+c . c .\right] \\
& +\left[i \omega_{p}\left(-\frac{\mu_{p}}{c} \frac{\partial}{\partial z}+\frac{\partial}{\partial t}\right) F_{b}(z, r, t) e^{-i \omega_{p} t} e^{i k_{p} \mu_{p} z}+c . c .\right],
\end{aligned}
$$

and in the paraxial approximation and approximation of a rotating wave for the spatial derivatives of Stokes standing waves, the following condition is satisfied, [10],

$$
\nabla^{2} E_{\text {Stokes }}(z, r, t)=-\frac{\mu_{S}(z)^{2}}{c^{2}} \frac{1}{2}\left(\omega_{S}^{2} E_{S}(t) e^{-i \omega_{S} t}+\text { c.c. }\right) U_{S}(r, z)
$$

and respectively,

$$
\nabla^{2} E_{\text {Stokes }}(z, r, t)-\frac{1}{c^{2}} \frac{\partial^{2}}{\partial t^{2}} E_{\text {Stokes }}(z, r, t)=\frac{\mu_{S}(z)^{2}}{c^{2}}\left(i \omega_{S} \frac{\partial E_{S}(t)}{\partial t} e^{-i \omega_{S} t}+c . c .\right) U_{S}(z, r) .
$$

Equations (8), (9) and (11) create the transformed left-hand side of the wave equation (10). The nonlinear polarization of the Raman crystal on the right-hand side of the wave equation (6) is determined by the fields of the pump and the Stokes component, by vibrations of the Raman centers in the Raman crystal, and is described by the Plaček formula [12]:

$$
P(z, r, t)=N\left(\frac{\partial \alpha}{\partial q}\right) q(z, r, t)\left[E_{\text {Stokes }}(z, r, t)+F_{\text {pump }}(z, r, t)+F_{\text {backward }}(z, r, t)\right],
$$

where $q(z, r, t)$ is the vibration amplitude of the RA-centers in the crystal, $N$ is the density of the RA-centers in the crystal, and $(\partial \alpha / \partial q)$ is the polarizability derivative of the Raman crystal in terms of the spatial coordinate.

Derivation and analysis of equations A1 to A20 (see Appendix, part VI) create the transformed right-hand side of the wave equation (6):

$$
\begin{aligned}
c & \frac{4 \pi}{c^{2}} \frac{\partial^{2}}{\partial t^{2}} P(z, r, t)=\frac{\pi}{c^{2}} \frac{1}{4 M} N\left(\frac{\partial \alpha}{\partial q}\right)^{2} \frac{T_{2}}{\omega_{0}} \\
& \times\left[i \omega_{S}^{2} e^{-i \omega_{S} t}\left(F_{P}^{*}(z, r, t) F_{P}(z, r, t)+F_{b}^{*}(z, r, t) F_{b}(z, r, t)\right) E_{S}(t) U_{S}(z, r)\right. \\
& \left.-i \omega_{P}^{2} e^{-i \omega_{P} t} F_{p}(z, r, t) E_{S}^{*}(t) E_{S}(t) U_{S}(z, r)^{2}-i \omega_{P}^{2} e^{-i \omega_{P} t} F_{b}(z, r, t) E_{S}^{*}(t) E_{S}(t) U_{S}(z, r)^{2}+c . c .\right] .
\end{aligned}
$$


As a result, the derivations of equations (8), (9), (11), and (13) yield a transformed wave equation not containing the temporal and longitudinal spatial derivatives of the second order. The wave equation for the amplitude of the Stokes component is obtained from equations (11) and (13) by selecting terms with a factor $e^{-i \omega_{s} t}$,

$$
\mu_{S}^{2} \frac{\partial E_{S}(t)}{\partial t} U_{S}(z, r)=\frac{\pi}{4 M} N\left(\frac{\partial \alpha}{\partial q}\right)^{2} \frac{\omega_{S}}{\omega_{0}} T_{2}\left(F_{P}^{*}(z, r, t) F_{P}(z, r, t)+F_{b}^{*}(z, r, t) F_{b}(z, r, t)\right) E_{S}(t) U_{S}(z, r) .
$$

Multiplying the left and right sides of equation (14) by $U_{S}(z, r)$ and integrating the equation over the volume of the cavity with allowance for equation (5), we obtain the equation:

$$
\frac{\partial E_{S}(t)}{\partial t}=g_{S} \frac{c^{2} \mu_{p}}{16 \pi L_{S}} E_{S}(t) \int_{V c r y s}\left(F_{p}^{*}(z, r, t) F_{p}(z, r, t)+F_{b}^{*}(z, r, t) F_{b}(z, r, t)\right) U_{S}^{2}(r, t) 2 \pi r d r d z,
$$

where $V_{\text {crys }}$ is the volume of a Raman crystal, and $g_{S}$ is the gain of SRS [12]

$$
g_{S}=\frac{4 \pi^{2} T_{2} \omega_{S} N}{\mu_{p} \mu_{S} c^{2} M \omega_{0}}\left(\frac{\partial \alpha}{\partial q}\right)^{2}
$$

The wave equations for the amplitudes of the pump forward and backward pulses are obtained from Eqs. (8), (9), (11) by selection of terms with the factor $e^{-i \omega_{p} t+i \mu_{p} k_{p} z}$, or $e^{-i \omega_{p} t+i \mu_{p} k_{p} z}$, respectively:

$$
\begin{aligned}
& \frac{1}{i} \frac{c^{2}}{\mu_{p}^{2} \omega_{p}} \frac{1}{2}\left(\frac{\partial^{2}}{\partial x^{2}}+\frac{\partial^{2}}{\partial y^{2}}\right) F_{p}(z, r, t)+\left(\frac{c}{\mu_{p}} \frac{\partial}{\partial z}+\frac{\partial}{\partial t}\right) F_{p}(z, r, t) \\
= & -g_{S} \frac{c^{2} \omega_{p}}{16 \pi \mu_{p} \omega_{0} \omega_{S}} F_{p}(z, r, t) E_{S}^{*}(t) E_{S}(t) U_{S}^{2}(r, t),
\end{aligned}
$$

and

$$
\begin{aligned}
& \frac{1}{i} \frac{c^{2}}{\mu_{p}^{2} \omega_{p}} \frac{1}{2}\left(\frac{\partial^{2}}{\partial x^{2}}+\frac{\partial^{2}}{\partial y^{2}}\right) F_{b}(z, r, t)+\left(-\frac{c}{\mu_{p}} \frac{\partial}{\partial z}+\frac{\partial}{\partial t}\right) F_{b}(z, r, t) \\
= & -g_{S} \frac{c^{2} \omega_{p}}{16 \pi \mu_{p} \omega_{0} \omega_{S}} F_{b}(z, r, t) E_{S}^{*}(t) E_{S}(t) U_{S}^{2}(r, t),
\end{aligned}
$$

where from the term $U_{S}^{2}(r, t)$ in equation (15), based on the approximation of the rotating wave, the term with high-frequency oscillation about zero is removed

$$
\begin{aligned}
U_{S}^{2}(r, t) & =\sin \left(k_{S}^{(m)}\left(z-z_{0}^{(m)}\right)\right)^{2} \frac{2}{L_{S} \mu_{S}} \frac{2}{\pi r_{S}(z)^{2}} \exp \left(-\frac{2 r^{2}}{r_{S}(z)^{2}}\right) \\
& =\left(\frac{1}{2}\right) \frac{2}{L_{S} \mu_{S}} \frac{2}{\pi r_{S}(z)^{2}} \exp \left(-\frac{2 r^{2}}{r_{S}(z)^{2}}\right) .
\end{aligned}
$$

If the diffraction of the Stokes component is negligible, then second-order derivatives in transverse coordinates can be neglected in Eq. (15). In this case, all the functions in equations (15), (17) and (18) will be real, which makes it possible to change from the amplitudes of the 
pump wave and the backward wave (for example, the pump wave reflected by the output mirror) to the intensities of these waves, $I_{p}(z, r, t)$, and, $I_{b}(z, r, t)$, respectively,

$$
\begin{aligned}
& I_{p}(z, r, t)=\frac{c \mu_{p}}{4 \pi} F_{p}^{*}(z, r, t) F_{p}(z, r, t), \\
& I_{b}(z, r, t)=\frac{c \mu_{p}}{4 \pi} F_{b}^{*}(z, r, t) F_{b}(z, r, t),
\end{aligned}
$$

and from the generated Stokes components to the Stokes energy $W_{S}$ in the resonator (see Eq. (5)). As a result, the equation for $W_{S}(t)$ in the resonator takes the form:

$$
\frac{\partial W_{S}(t)}{\partial t}=\frac{c g_{S}}{8 L_{S}} W_{S}(t) \int_{V c r y s}\left(I_{p}(r, z, t)+I_{b}(r, z, t)\right) \frac{4 r}{r_{S}(z)^{2}} \exp \left(-\frac{2 r^{2}}{r_{S}(z)^{2}}\right) d r d z
$$

and the equations for intensities of the forward and backward pump waves read as:

$$
\begin{gathered}
\left(\frac{c}{\mu_{p}} \frac{\partial}{\partial z}+\frac{\partial}{\partial t}\right) I_{p}(z, r, t)=-g_{S} \frac{c^{2} \omega_{p}}{4 L_{S} \mu_{p} \omega_{S}} \frac{2}{\pi r_{S}(z)^{2}} \exp \left(-\frac{2 r^{2}}{r_{S}(z)^{2}}\right) I_{p}(z, r, t) W_{S}(t) . \\
\left(-\frac{c}{\mu_{p}} \frac{\partial}{\partial z}+\frac{\partial}{\partial t}\right) I_{b}(z, r, t)=-g_{S} \frac{c^{2} \omega_{p}}{4 L_{S} \mu_{p} \omega_{S}} \frac{2}{\pi r_{S}(z)^{2}} \exp \left(-\frac{2 r^{2}}{r_{S}(z)^{2}}\right) I_{b}(z, r, t) W_{S}(t) .
\end{gathered}
$$

Eqs. (22), (23) and (24) have been used for modeling of Stokes generation dynamics in ECRL. The space-time dependence of the pump intensity in experimental pulses is approximated by the expression.

The space-time dependence of the pump intensity in experimental pulses is approximated by the expression

$$
I_{p}(z, r, t)=W_{p} \frac{2}{\pi r_{p}^{2}} \exp \left(-\frac{2 r^{2}}{r_{p}^{2}}\right) \sqrt{\frac{2}{\pi}} \frac{1}{\tau} \exp \left(-\frac{2\left(\left(t-t_{0}\right)-\frac{1}{c}\left(z-z_{0}\right)\right)}{\tau^{2}}\right),
$$

where $\tau=\sqrt{\tau_{p} / 2 \ln (2)}, z_{0}$ is the coordinate of the input face of the Raman crystal, and $t_{0}$ is the "start" simulation time.

In the laser, the output mirror reflects the pump beam, and the reflected beam becomes the inverse of the pump beam. As a result, for the intensities $I_{p}\left(z_{m}, r, t\right)$ and $I_{b}\left(z_{m}, r, t\right)$ where $z_{m}$ is the longitudinal coordinate of the output mirror, a boundary condition is created, written down by the algebraic equation

$$
I_{b}\left(z_{m}, r, t\right)=m_{p, \text { out }} I_{f}\left(z_{m}, r, t\right) .
$$

Thus, a system of differential and algebraic equations (22), (23), (24), (25) and (26) describing the generation and dynamics of Raman laser radiation has been developed.

\section{EXPERIMENT}

A principal optical scheme of the experimental setup is shown in Fig. 1. The pump laser was a diode-pumped frequency-doubled actively-Q-switched Nd:YAG laser. The laser generated multimode pulses at $532 \mathrm{~nm}$ with the full spectral width of $0.7 \mathrm{~cm}^{-1}$ at half the maximum and a pulse energy up to $W_{p}=100 \mathrm{~mJ}$ at the repetition rate of $10 \mathrm{~Hz}$. A typical laser pulse was slightly asymmetric in its shape and considerably structured as the temporal measurements were revealed 
(Fig. 2a). The intergrated pulse width $\tau_{p}$ of a cumulative measurement over $10^{3}$ laser shots oscilloscope trace (Fig. 2b) was measured to be about $7 \mathrm{~ns}$ (FWHM). The linearly polarized pump pulse beam possessed angular divergence of 0.7 and $0.5 \mathrm{mrad}$ in horizontal and vertical planes, respectively. This beam was compressed down to $2 r_{p} \sim 4 \mathrm{~mm}$ in diameter, collimated, and then directed into the cavity of ECRL.

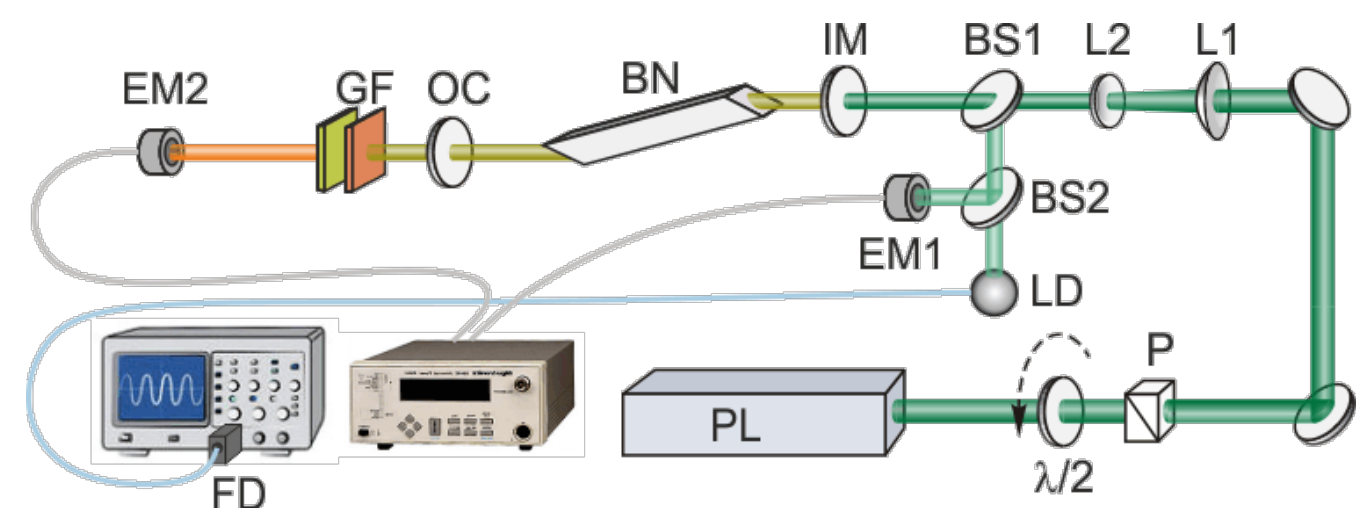

Fig. 1. Principal scheme of the experimental setup: PL: frequency-doubled Nd:YAG pump laser; $\lambda / 2$ : half-wave plate at the $532 \mathrm{~nm}$ wavelength; $\mathrm{P}$ : polarizer; $\mathrm{L} 1,2$ : positive and negative lenses of the pump beam compressor; BS1,2: beam splitters; IM: input mirror of the Raman cavity; BN: barium nitrate crystal; OC: output coupler of the Raman cavity; GF: color glass filters; LD: light diffuser; EM1,2: sensors of the energy meter; FD: fast photodetector.
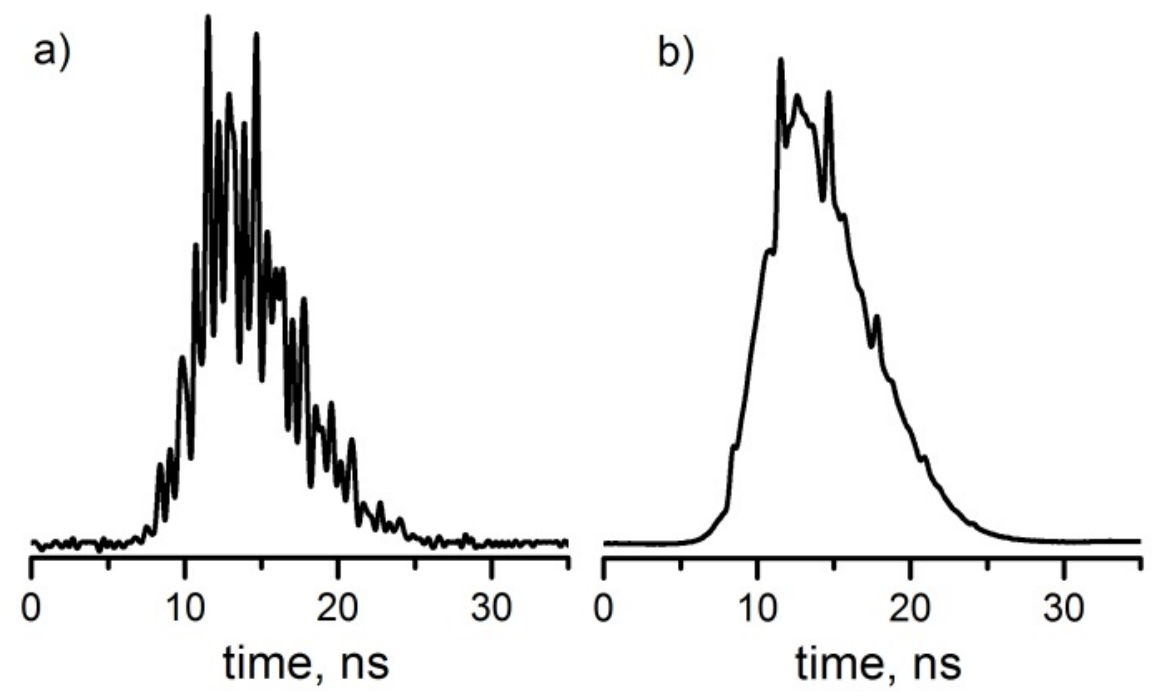

Fig. 2. Typical single-shot (a) and cumulative over $10^{3}$ laser shots (b) oscilloscope traces of the pump pulses. 
The Raman laser cavity was formed by two concave dichroic mirrors located at distance of $110 \mathrm{~mm}$ one from another. The input mirror with a curvature radius of $R_{\text {in }}=-767 \mathrm{~mm}$ was relatively transparent at the pump radiation wavelength. High reflection at the pump wavelength of the output coupler with its curvature radius of $R_{\text {out }}=-737 \mathrm{~mm}$ provided the double-pass pump configuration. Coefficients of reflection for the input mirror $m_{p, i n}, m_{S, i n}$ and output coupler $m_{p, \text { out }}$, $m_{S, \text { out }}$ at the pump and $1^{\text {st }}$ Stokes wavelengths are listed in Table 1.

Table 1. Parameters of ECRL studied.

\begin{tabular}{lll}
\hline \hline pump wavelength, $\mathrm{nm}$ & $\lambda_{p}$ & 532 \\
\hline Stokes wavelength, $\mathrm{nm}$ & $\lambda_{S}$ & 563 \\
\hline pump beam radius, $\mathrm{mm}$ & $r_{P}$ & 0.8 \\
\hline Stokes beam radius, mm & $r_{0}$ & 0.5 \\
\hline pump pulse duration at half the maximum, ns & $\tau_{p}$ & 7 \\
\hline "start" calculation time, $\mathrm{ns}$ & $t_{0}$ & $-2 \tau_{p}$ \\
\hline Raman crystal length, mm & $L_{c r}$ & 67 \\
\hline refractive index of Raman crystal at $\lambda_{p}$ & $\mu_{p}$ & 1.5768 \\
\hline refractive index of Raman crystal at $\lambda_{S}$ & $\mu_{S}$ & 1.5736 \\
\hline Raman gain coefficient at $\lambda_{p}$, cm / GW & $g_{S}$ & 48.4 \\
\hline reflection coefficient of the input mirror at $\lambda_{p}$ & $m_{p, \text { in }}$ & 0.12 \\
\hline reflection coefficient of the output coupler at $\lambda_{p}$ & $m_{p, \text { out }}$ & 0.9996 \\
\hline reflection coefficient of the input mirror at $\lambda_{S}$ & $m_{S, \text { in }}$ & 0.99 \\
\hline reflection coefficient of the output coupler at $\lambda_{S}$ & $m_{S, \text { out }}$ & 0.25 \\
\hline \hline
\end{tabular}

Raman frequency conversion was observed in a crystal of barium nitrate $(\mathrm{BN})$ where the most intense Raman-active phonon mode oscillated with frequency of $31.4 \mathrm{THz}$ [13]. At the pump wavelength $\lambda_{p}=532 \mathrm{~nm}$, this enabled the $1^{\text {st }}$ Stokes wavelengths $\lambda_{S}=563 \mathrm{~nm}$. The BN crystal with the Brewster-cut ends had the length $L_{c r}=67 \mathrm{~mm}$ and transverse dimensions $7 \times 10 \mathrm{~mm}$ in vertical and horizontal directions, respectively. This BN crystal was mounted into a special oven where its temperature was maintained at $\sim 50^{\circ} \mathrm{C}$. Stokes radiation generated in the $\mathrm{BN}$ was spectrally separated from residual pump radiation by color-glass filters placed after the output coupler of the Raman cavity.

Energy of the pump and Stokes pulses was controlled by pyroelectric detectors Ophir PE-9 and PE-10 though Ophir Pulsar2 interface. Two fast photodetectors (Thorlabs SV2-FC with the rise/fall time of $150 \mathrm{ps}$ ) and a digital oscilloscope (Tektronix TDS5104) possessing the transmission bands of $1 \mathrm{GHz}$ were used to render laser pulse profiles. Fabry-Perot interferometer with the free-spectral range of $2.5 \mathrm{~cm}^{-1}$ was taken to spectral measurement. 


\section{RESULTS AND DISCUSSION}

The experimentally measured dependence of the Stokes pulse energy on the pump energy is shown in Fig. 3 (hollow dots). In the experiment, we have observed the Raman threshold if $W_{p}=16 \mathrm{~mJ}$. Energy of the Stokes pulses has been measured to be $W_{S}=28 \mathrm{~mJ}$ corresponding to a pump-to-Stokes conversion efficiency $W_{S} / W_{p}=29 \%$ at the pump energy $W_{p}=95 \mathrm{~mJ}$. As is seen, a slop of the experimental dependence gradually decreased for relatively high pump energy. This should be attributed to depletion of the $1^{\text {st }}$ Stokes pulses due to the $2^{\text {nd }}$ and higher-order Stokes excitation for which generation thresholds were observed at the pump energy $W_{p}>50 \mathrm{~mJ}$.

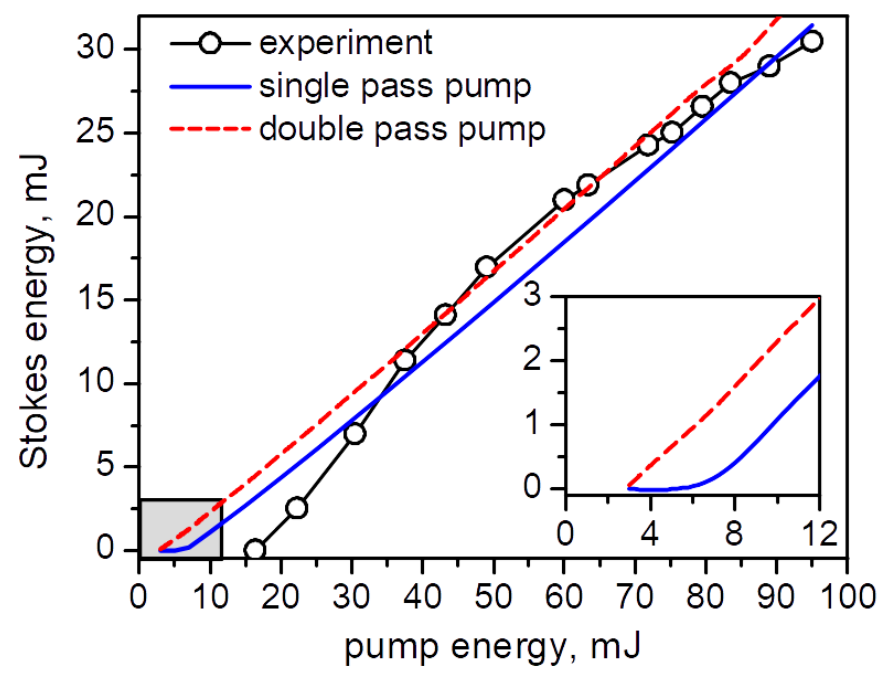

Fig. 3. Experimentally measured (hollow dots) and numerically calculated for singlepass (blue solid line) and double- pass (red dashed line) pump configurations Stokes energy as a function of the pump energy.

The numerically calculated dependences of the Stokes energy as a function of the pump energy are shown in Fig. 3 for the single- pass (blue solid line) and double- pass (red dashed line) pump configurations. Single-pass means that the pump beam has only a single pass through the Raman crystal and then goes output by the output mirror. Double-pass means a double passage of the pump beam through the Raman crystal due to a significant reflection of the pump beam by the output coupler. We have written explicitly Equation (26) to describe the reflection of the pump radiation from the output mirror. The important difference between the single-pass and double-pass is that the double-pass configuration leads to a decrease in the Raman laser threshold and to an increase in the energy of the Stokes pulse. Parameters of the simulation were ported to the model from the experiment and their list is shown in Table 1. As is seen, the theoretical data reproduced well experimental results when the pump energy $W_{p}$ was higher than $35 \mathrm{~mJ}$. Herewith, the dependencies for the single-pass and double-pass pump configurations demonstrate that the slopes are nearly equal and locate relatively close to each other. Such a behaviour becomes clear if we take into account the same intra-cavity losses at the Stokes wavelength in the two configurations. 
The only noticeable difference between the theoretical and experimental data was observed at low $W_{p}$ values. Thus, the experimentally measured generation threshold of the ECRL was several times higher than that given by the theory. More specifically, the numerical data revealed the Raman laser thresholds at $W_{p} \approx 7 \mathrm{~mJ}$ and $W_{p}=3 \mathrm{~mJ}$ for the single-pass and double-pass pump configuration, respectively (see the inset in Fig. 3). We attributed the near-threshold deviations between the theoretical and experimental energy dependencies to the laser beams astigmatism inside the Raman medium. Such an astigmatism originates from non-orthogonal incidence of the laser beams onto the ends of the BN crystal which were cut under Brewster angle with respect to the light propagation direction. Our theory considers axisymmetric interaction geometry and does not take into account the laser beam astigmatism.

The program for numerical solution of equations (22), (23), (24), (25) and (26) is designed so that during the solution the time dependences of the pump pulse intensities are numerically determined and used in the calculations at the transverse distance $R_{\text {offset }}$ from the optical axis of the beam in the interval from zero to a few radii $r_{0}$. Figure 4 shows intensity profiles of the intracavity pump pulse at the Raman crystal output calculated for the single-pass pump configuration at different distances $R_{\text {offset }}$ when these distances were shorter (Fig. 4a) and longer (Fig. 4b) than the Stokes beam radius $r_{0}$. Energy of the input pump pulse was taken to be $W_{p}=50 \mathrm{~mJ}$. The pump intensity profiles features asymmetry in position of the depletion region that can be attributed to the optical feedback effect. When $R_{\text {offset }}<r_{0}$, the pump intensity depletes to zero. The depletion appears almost simultaneously at different $R_{\text {offset }}$ from the beam axis. In the opposite situation, when $R_{\text {offset }}>r_{0}$, a level of the depletion gradually decrease with displacement from the beam axis. For $R_{\text {offset }}>1.6 r_{0}$, no evident depletion of the pump intensity is observed.
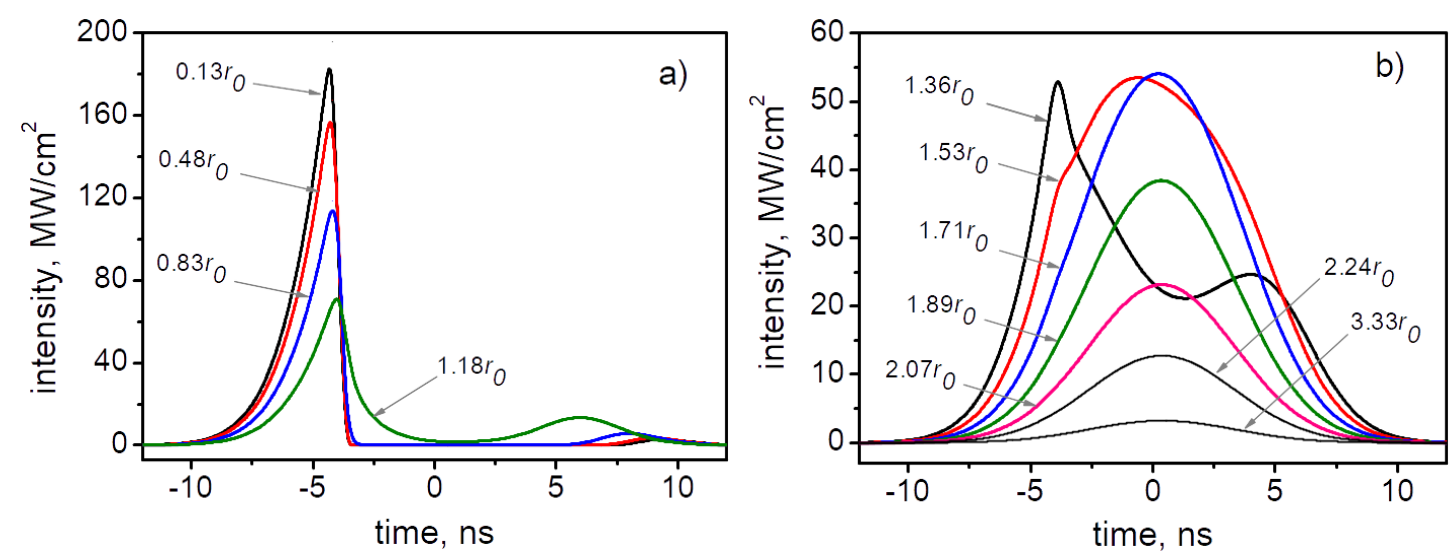

Fig. 4. The single-pass pump configuration: intensity versus time profiles of the depleted pump pulse issued from numerical calculations for different displacements from the beam optical axis.

The intensity profiles of the backward-reflected pump pulses which have made two passes through the Raman crystal are shown in Fig. 5a for the double - pass pump configuration and for the same energy of the input pump pulse. As compared to the single-pass data (Fig. 4a), here we see nearly twice decrease in the pump intensity for which the depletion develops. Specifically, in the first case, the depletion appears if the near-axial pump intensity approaches to $200 \mathrm{MW} / \mathrm{cm}^{2}$, 
whereas in the second case, the pump intensity does not exceed $110 \mathrm{MW} / \mathrm{cm}^{2}$. This clearly demonstrates the benefits of the double-pass pump configuration that is capable to reduce considerably the Raman laser threshold. We also have found that the double-pass pump configuration promoted the Stokes energy gain by 3-5 $\mathrm{mJ}$ with respect to the single-pass one.

An integral power profile of the corresponding Stokes pulse shown in Fig. 5b is nearly Gaussian which is the profile of the input pump pulse in our model. The Stokes pulse duration (6.85 ns) approaches to the pump pulse duration. These are surprising results if referred to the energy conversion efficiency of the pump beam into the Stokes beam which was only $33 \%$ for this case. They can be explained by the fact that energy of only paraxial part of the pump beam is efficiently converted to the Stokes radiation.

The numerical data presented above have been obtained when the Stokes mode radius $r_{0}=$ $0.5 \mathrm{~mm}$ was taken to be somewhat less than the radius of the pump beam $r_{p}=0.8 \mathrm{~mm}$. For these conditions, we have observed the best correspondence with the experiment. However, our theoretical data indicated the highest conversion efficiency of $40 \%$ at the same pump energy when $r_{0}$ was equal to $r_{p}$. We attribute this discrepancy to the effect of parametric four-wave processes and higher-order Stokes excitation which were not taken into account by our model.
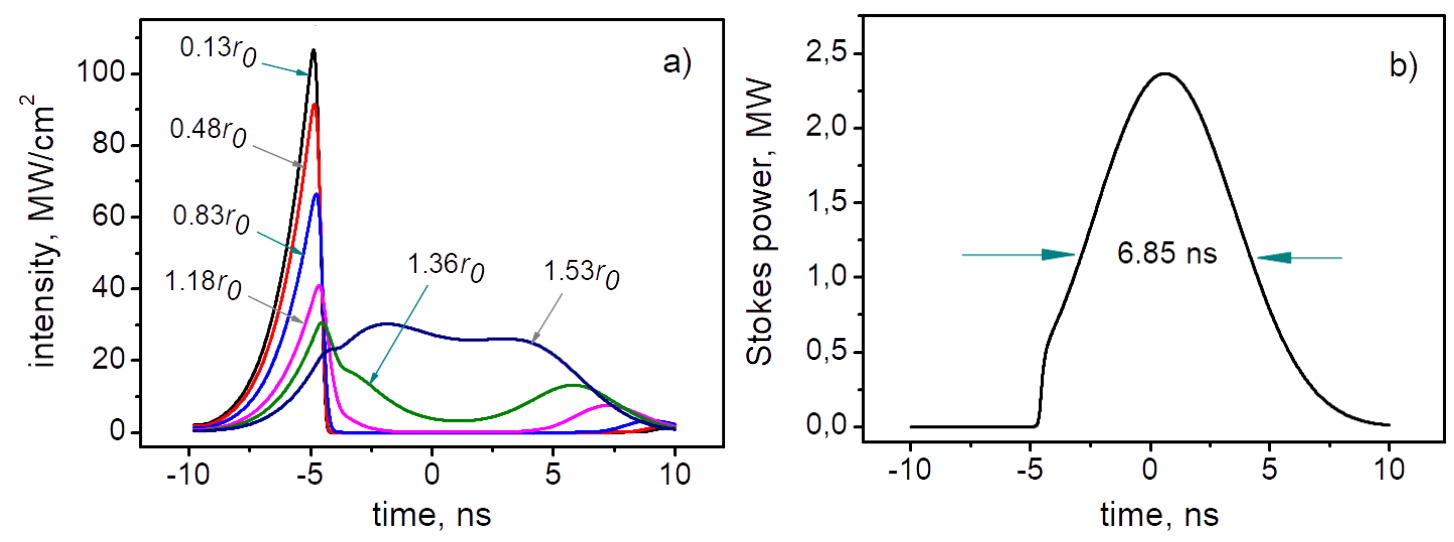

Fig. 5. The double-pass pump configuration: (a) intensity profiles of the depleted pump pulse for different displacement from the beam optical axis; (b) power profile of the corresponding Stokes pulse.

\section{CONCLUSION}

The semiclassical theoretical 3-D model of the external cavity Raman laser (ECRL) with a singly resonant cavity has been developed. In such an ECRL, cavity is resonant at the Stokes wavelength and not resonant at the pump wavelength. The wave equations for the amplitudes of the pump and Stokes pulses have been derived. The derivation has been performed under the rotating wave, slowly varying envelope and paraxial approximations which allowed transformation of the terms with the second-order spatial and temporal derivatives into terms with longitudinally spatial and temporal derivatives of the first order.

Obtained results of the theoretical modeling have been analyzed for our experimental ECRL conditions. The external cavity Raman laser on barium nitrate crystal has been pumped by the 
pulses at $532 \mathrm{~nm}$ from the actively Q-switched frequency-doubled Nd:YAG laser. We have shown that theoretically calculated energy characteristics of the Raman laser and space-time dynamics of the Stokes generation were in reasonable agreement with the experimentally obtained ones.

\section{ACKNOWLEDGEMENTS}

The authors are grateful to Prof. Nguyen Dai Hung for useful discussions. This research was supported by Belarusian Republican Foundation for Fundamental Research (Project $\Phi$ 17B006) and Vietnam Academy of Science and Technology (Project VAST.HTQT.Belarus.04/17-18).

\section{REFERENCES}

[1] V. A. Lisinetskii, Th. Riesbeck, H. Rhee, H.J. Eichler, V.A. Orlovich. Appl. Phys. B 99 (2010) 127

[2] P. Cerny, Helena Jeliınkova, P.G. Zverev, T.T. Basiev. Progress in Quantum Electronics 28 (2004) 113.

[3] A. A. Demidovich, P. A. Apanasevich, L. E. Batay, A. S. Grabtchikov, A. N. Kuzmin, V. A. Lisinetskii, V. A. Orlovich, O.V. Kuzmin, V. L. Hait, W. Kiefer, M.B.Danailov. J. Opt. Soc. Am. B 23 (2006) 1109.

[4] R. V. Chulkov, A. S. Grabtchikov, D.N. Busko, P.A. Apanasevich, N. A. Khilo, and V.A. Orlovich. J. Opt. Soc. Am. B 23 (2006) 1109.

[5] E. Berike, B. Davidenko, V. Mihkelsoo, P. A. Apanasevich, A. S. Grabtchenko, V.F. Orlovich. Opt. Commun. 56 (1985) 283

[6] A. A. Demidovich, A. S. Grabtchikov, V. A. Lisinetskii, V.N.Burakevich V.A. Orlovich, W.Kiefer. Opt. Lett., 30 (2005) 1701.

[7] A. S. Grabtchikov, R. V. Chulkov, V. A. Orlovich, M. Schmitt, R. Maksimenko, W. Kiefer. Opt. Lett., 28 (2003) 926.

[8] S. V. Voitikov, A. A. Demidovich, P. V. Shpak, A. S. Grabtchikov, M. B. Danailov, and V. A. Orlovich. J. Opt. Soc. Am. B 27 (2010) 1232.

[9] H. Haken. Laser Light Dynamics, Elsevier Science Publishers B. V (1985)

[10] A. E. Siegman. Lasers, University Science Books, (1986)

[11] S. V. Voitikov, A. A. Demidovich, M.B. Danailov, and V.A. Orlovich. Journal of Physics B: At. Mol. Opt. Phys. 47 (2014) 105402

[12] A. Penzkofer, A.Laubereau, W.Kaiser. Progress in Quantum Electronics 6 (1979) 55

[13] P. G. Zverev, T. T. Basiev, V. V. Osiko, A. M. Kulkov, and V. N. Voitsekhovskii. Opt. Materials. 11 (1999) 315. 


\section{APPENDIX}

According to Equation (12) the equation for the vibrational amplitude of the RA-centers is

$$
\begin{aligned}
& \frac{\partial^{2}}{\partial t^{2}} q(z, r, t)+\frac{2}{T_{2}} \frac{\partial}{\partial t} q(z, r, t)+\omega_{0}^{2} q(z, r, t) \\
= & \frac{1}{2 M}\left(\frac{\partial \alpha}{\partial q}\right)\left[E_{\text {Stokes }}(z, r, t)+F_{\text {pump }}(z, r, t)+F_{\text {backward }}(z, r, t)\right]^{2},
\end{aligned}
$$

where $T_{2}$ is the transverse relaxation time of the oscillations of the RA-centers, $M$ is the mass of the RA-centers, and $\omega_{0}$ is the frequency of the natural vibrations of the RA-centers. On the basis of the approximation of slowly varying amplitudes, the amplitude of the oscillations of the RA-centers is approximated by the expression

$$
q(z, r, t)=\frac{1}{2}\left[q_{0}(z, r, t) e^{-i \omega_{0} t}+c . c .\right],
$$

where $q_{0}(z, r, t)$ is the slowly varying amplitude of the vibrations of the RA- centers, and the lefthand side of equation (A.1) with the derivative of the second order is transformed into expression

$$
\begin{aligned}
& \frac{\partial^{2}}{\partial t^{2}} q(z, r, t)+\frac{2}{T_{2}} \frac{\partial}{\partial t} q(z, r, t)+\omega_{0}^{2} q(z, r, t) \\
= & \frac{\omega_{0}}{i}\left[\frac{\partial}{\partial t} q_{0}(z, r, t) e^{-i \omega_{0} t}+\frac{1}{T_{2}} q_{0}(z, r, t) e^{-i \omega_{0} t}\right]+c . c .,
\end{aligned}
$$

and the right-hand side of equation (A.1) is transformed into the expression

$$
\begin{aligned}
& \frac{1}{2 M}\left(\frac{\partial \alpha}{\partial q}\right)\left[E_{\text {Stokes }}(z, r, t)+F_{\text {pump }}(z, r, t)+F_{\text {backward }}(z, r, t)\right]^{2} \\
= & \frac{1}{4 M}\left(\frac{\partial \alpha}{\partial q}\right)\left(F_{p}(z, r, t)+F_{b}(z, r, t)\right) e^{-i \omega_{p} t} E_{S}^{*}(z, r, t) e^{i \omega_{S} t} U_{S}(z, r)+c . c .
\end{aligned}
$$

Thus, the transformed equation for the amplitude of local vibrations of RA-centers has the form

$$
\begin{aligned}
& {\left[\frac{\partial}{\partial t} q_{0}(z, r, t) e^{-i \omega_{0} t}+c . c .+\frac{1}{T_{2}} q_{0}(z, r, t) e^{-i \omega_{0} t}\right]+c . c . } \\
= & \frac{i}{\omega_{0}} \frac{1}{4 M}\left(\frac{\partial \alpha}{\partial q}\right)\left(F_{p}(z, r, t)+F_{b}(z, r, t)\right) e^{-i \omega_{p} t} E_{S}^{*}(z, r, t) e^{i \omega_{S} t} U_{S}(z, r)+c . c .
\end{aligned}
$$

When pumped by nanosecond pulses and the rate of change in the amplitude of the oscillations is substantially less than $1 / T_{2}$ and

$$
\left|\frac{\partial}{\partial t} q_{0}(z, r, t)\right| \ll \frac{1}{T_{2}}\left|q_{0}(z, r, t)\right|,
$$

the solution of equation (A.5) is simplified, and the amplitude of local oscillations of RA-centers is:

$$
q_{0}(z, r, t)=\frac{i T_{2}}{\omega_{0}} \frac{1}{4 M}\left(\frac{\partial \alpha}{\partial q}\right)\left(F_{p}(z, r, t)+F_{b}(z, r, t)\right) E_{S}^{*}(t) U_{S}(z, r),
$$

which makes it possible to write down an expression for the nonlinear induced polarization of a RA-medium. In the framework of the paraxial approximation and the approximations of a rotating 
wave and slowly varying amplitudes and under conditions when the rate of change in the amplitude of the vibrations of the RAcenters is much less than $1 / T_{2}$, the induced polarization of the RAmedium is

$$
\begin{aligned}
P(z, r, t)= & N\left(\frac{\partial \alpha}{\partial q}\right)\left(\frac{1}{4} q_{0}^{*}(z, r, t) e^{-i\left(\omega_{P}-\omega_{0}\right) t} F_{p}(z, r, t)+c . c .\right) \\
= & \frac{1}{16 M} N\left(\frac{\partial \alpha}{\partial q}\right)^{2} \frac{T_{2}}{\omega_{0}}\left[-i e^{-i \omega_{S} t}\left(F_{P}^{*}(z, r, t) F_{P}(z, r, t)+F_{b}^{*}(z, r, t) F_{b}(z, r, t)\right) E_{S}(t) U_{S}(z, r)\right. \\
& \left.+i e^{-i \omega_{P} t} F_{p}(z, r, t) E_{S}^{*}(t) E_{S}(t) U_{S}(z, r)^{2}+i e^{-i \omega_{p} t} F_{b}(z, r, t) E_{S}^{*}(t) E_{S}(t) U_{S}(z, r)^{2}+c . c .\right],
\end{aligned}
$$

which allows to obtain the formula for the right-hand side of the wave equation (6). 